\section{Revista dePolítica Económica y Desarrollo Sostenible}

Centro Internacional de Política Económica para el Desarrollo Sostenible
Revista de Política Económica y Desarrollo Sostenible

EISSN: 2215-4167 • Vol. 4 (1) • Julio-Diciembre, 2018: 1-20

DOI: http://dx.doi.org/10.15359/peds.4-1.2

URL: http://www.revistas.una.ac.cr/politicaeconomica

Revista electrónica semestral publicada por el Centro Internacional de Política Económica para el Desarrollo Sostenible

Universidad Nacional, Lagunilla, Heredia Apartado 2393-3000

\title{
Innovación y crecimiento inclusivo en Costa Rica: el caso del sector lácteo
}

\section{Innovation and inclusive growth in Costa Rica: the case of dairy sector \\ Jeffrey Orozco-Barrantes ${ }^{1}$, Luis M. Barboza-Arias ${ }^{2}$}

Fecha de recibo: 28 de junio del 2018. Fecha de corrección: 31 de julio del 2018.

Fecha de aceptación: 1 de octubre del 2018. Fecha de publicación: 22 de octubre del 2018

\begin{abstract}
Resumen
El propósito de este artículo es analizar la estructura del sistema de innovación agropecuario y su relación con el crecimiento inclusivo en el sector lácteo de Costa Rica. El marco conceptual que se utiliza incorpora tres conceptos principales: el desarrollo sostenible, los sistemas de innovación y el crecimiento inclusivo. Sugerimos la articulación de estos conceptos en un marco analítico conjunto con la finalidad de entender el tipo de interacciones que se establecen entre el desarrollo de las innovaciones y el crecimiento inclusivo a nivel sectorial. La ganadería de leche se ha visto afectada en los últimos veinticinco años por cambios en la producción primaria y el mercado. En consecuencia, la planificación de procesos para la innovación ha sido el resultado de un conjunto de dinámicas históricas y organizacionales fuertemente influenciadas por la participación de las cooperativas y sus objetivos de desarrollo productivo. Se identifican al menos cuatro ejes prioritarios para la gestión de la innovación y la competitividad del sector: mejoramiento genético, nutrición, manejo de pasturas y adaptación al cambio climático. Concluimos que cualquier esfuerzo para estimular la innovación y el conocimiento en la cadena láctea, sea este de carácter institucional o apoyado desde la política pública debe: corresponderse con los objetivos de la política de desarrollo nacional, contener una perspectiva de desarrollo territorial y dialogar de modo reflexivo con los diferentes actores que intervienen en la gestión del aprendizaje y el establecimiento de sinergias para la acción colectiva.
\end{abstract}

Palabras clave: Agroindustria, desarrollo sostenible, economía, industria lechera, producto agrícola.

\begin{abstract}
The purpose of this article is to analyze the structure of the agricultural innovation system and its contribution to inclusive growth in the dairy sector of Costa Rica. The conceptual framework incorporates three main concepts: sustainable development, innovation systems and inclusive growth. Milk cattle ranching has been affected in the last twenty-five years by changes in primary production and the market. Consequently, the planning of processes for innovation has been the result of a set of historical and organizational dynamics strongly influenced by the participation of cooperatives and their productive development objectives. At least four priority axes are identified for the management of innovation and the competitiveness of the sector: genetic improvement, nutrition, pasture management and adaptation to climate change. We conclude that any effort to stimulate innovation and knowledge in the dairy value chain, should: (1) correspond to the objectives of the national development policy, (2) contain a perspective of territorial development and (3) consider reflective dialogue processes with the different actors in the management of learning and the establishment of synergies for collective action.
\end{abstract}

Keywords: Agroindustry, dairy industry, economics, sustainable development, agricultural products.

1 .Economista, docente e investigador. Es actualmente el Director General del Centro Internacional de Política Económica, de la Universidad Nacional, jeffrey.orozco.barrantes@una.ac.cr.

2 .Investigador del Centro Internacional de Política Económica, de la Universidad Nacional. 
Revista de Política Económica • EISSN: 2215-4167

Vol. 4 (1) • Julio-Diciembre, 2018: 1-20

DOI: http://dx.doi.org/10.15359/peds.4-1.2

Orozco-Barrantes y Barboza-Arias

URL: http://www.revistas.una.ac.cr/politicaeconomica

\section{Introducción}

En este artículo se analiza la estructura del sistema de innovación agropecuario y su relación con el crecimiento inclusivo en el sector lácteo de Costa Rica. Se propone la utilización de un marco conceptual que articula tres conceptos principales: el desarrollo sostenible (Van den Bergh, 1996; Orozco, 2004), los sistemas de innovación (Edquist, 1997; Edquist y Johnson, 1997; Lundvall y Lorenz, 2010) y el crecimiento inclusivo (Ali y Zhuang 2007), con la finalidad de entender mejor el tipo de interacciones que se establecen entre el desarrollo de las innovaciones y el crecimiento inclusivo a nivel sectorial.

Nuestro argumento central es que el concepto de desarrollo sostenible da una idea clara de la naturaleza multidimensional de los retos que presenta el desarrollo. No obstante, para hacer más explícita la dimensión social se introduce el concepto de crecimiento inclusivo, abordándolo en el marco de la discusión sobre innovación y desarrollo inclusivo (Arocena y Sutz, 2002; Sampedro y Díaz, 2016).

Se asume que es pertinente analizar los retos del sector a partir de un enfoque multidimensional, por lo tanto, son de relevancia no solo los objetivos económicos, sino también los ambientales y sociales. De manera concreta, se analiza el proceso evolutivo de las transformaciones en el sector lácteo mediante un análisis del sistema de innovación. Además, se hace referencia al concepto de cadena global de mercancías (Kaplinsky, 2015; Gereffi y Lee, 2016), ya que esas cadenas pueden promover o limitar la capacidad de innovación de una empresa (Bijman y Ton, 2008).

Como en muchos países, el sector lácteo en Costa Rica tiene una serie de mecanismos de protección a los mercados internacionales. Sin embargo, en los tratados de libre comercio más importantes para la economía del país se contemplan procesos de apertura que han obligado a los actores del país a una dinámica de fortalecimiento competitivo cada vez más sólida. Algunos de los arreglos institucionales del pasado podrían seguir siendo de utilidad, pero otros probablemente tengan que adecuarse para seguir promoviendo un buen desempeño y cumplir los objetivos de crecimiento inclusivo (Arond et al., 2011).

El documento se estructura de la siguiente manera: la siguiente sección detalla el marco teórico que orienta la investigación. Más adelante, en la sección 3, el documento presenta la metodología utilizada y el estudio de caso. En la sección 4 se presenta los principales resultados y la discusión. Finalmente, se concluye con reflexiones e implicaciones para la teoría y el diseño de políticas.

\section{Marco teórico}

\subsection{Retos multidimensionales: el concepto de desarrollo sostenible}

El concepto de desarrollo sostenible se incorpora en el análisis dada la necesidad de tomar en cuenta diversos tipos de retos, incluyendo, entre otros, los económicos, sociales y ambientales, así como las contradicciones entre estos. 
Lo más relevante de la incorporación de este concepto en el análisis se centra en el objetivo de influir en la orientación política para lograr las transformaciones necesarias. Es decir, alcanzar una situación en que la explotación de los recursos, la dirección de las inversiones, la orientación del desarrollo tecnológico y los cambios institucionales se realicen de acuerdo con un desarrollo sostenible con cada vez menor desigualdad (Van den Bergh, 1996).

Originalmente, este concepto se propuso como un punto de partida para buscar soluciones a una serie de retos pendientes, así como para prevenir el surgimiento de conflictos potenciales asociados a las tensiones entre grupos de interés, clasificados en cuatro grupos. Entre los desafíos se encuentran: aumentar o maximizar el bienestar humano de las generaciones actuales, mantener suficientes oportunidades para garantizar el bienestar de las generaciones futuras, conservar o mejorar la calidad ambiental y la disponibilidad de los recursos naturales, y preservar la diversidad biótica y genética.

El concepto pretende dar solución al reto de resolver de manera simultánea los problemas de escala, distribución, equidad y ajuste, mediante la inversión y el avance tecnológico (Van den Bergh, 1996). Para el estudio de las innovaciones en sectores particulares, el concepto de desarrollo sostenible puede ser de gran utilidad, en tanto que introduce la idea de considerar la orientación y la velocidad del cambio tecnológico y la necesidad de hacer innovaciones institucionales, todas orientadas a lograr los objetivos en las diferentes dimensiones del desarrollo.

Como se sugiere en Orozco (2004), es importante considerar los retos multidimensionales a partir de un conjunto de indicadores y analizar cómo los procesos de innovación van generando o no mejoras en el desempeño medido mediante ese grupo de indicadores.

\subsection{Crecimiento inclusivo: la dimensión social de sostenibilidad}

Para abordar la dimensión social en el concepto de desarrollo sostenible, proponemos el concepto de crecimiento inclusivo. La discusión ha utilizado otros conceptos, como innovación inclusiva o desarrollo inclusivo, como se verá más adelante, pero en términos prácticos preferimos el concepto de crecimiento inclusivo.

De acuerdo con Ali y Zhuang (2007, p. 10):

El crecimiento inclusivo se refiere a un crecimiento con igualdad de oportunidades. Por lo tanto, el crecimiento inclusivo se enfoca tanto en crear oportunidades como en ponerlas al alcance de todos los individuos involucrados en una actividad económica específica. El crecimiento es inclusivo cuando se les permite a todos los miembros de una sociedad contribuir y participar en el proceso de crecimiento en igualdad de condiciones, independientemente de sus circunstancias individuales.

Una de las razones principales por las que los sistemas de innovación representan un enfoque válido para el estudio de los procesos de innovación en países en desarrollo es su pertinencia 
Revista de Política Económica • EISSN: 2215-4167

Vol. 4 (1) • Julio-Diciembre, 2018: 1-20

DOI: http://dx.doi.org/10.15359/peds.4-1.2

Orozco-Barrantes y Barboza-Arias

URL: http://www.revistas.una.ac.cr/politicaeconomica

metodológica para identificar el abordaje de la innovación desde espacios tradicionales que están fuera del radar de los enfoques clásicos (Chaminade et al., 2009).

Por lo tanto, la innovación para el desarrollo inclusivo debe verse como un medio de acceso a nuevos estadios del desarrollo, lo cual implica la búsqueda de soluciones novedosas a los problemas de pobreza, marginalidad y desigualdad, que operan como formas de exclusión tanto social como económica (Sampedro y Díaz, 2016).

Para el caso particular de actividades productivas que son menos intensivas en el uso de recursos tecnológicos, como ha sido la experiencia histórica de las actividades agropecuarias en la mayoría de países de América Latina, la discusión sobre la relación entre procesos de innovación e inclusión social adquiere no solo una mayor vigencia, sino que también invita a reflexionar sobre la necesidad de abordar el marco institucional desde el cual se articulan las políticas públicas y las estructuras de apoyo para este sector de forma sistemática.

Considerado desde esta perspectiva, el crecimiento inclusivo incorpora un conjunto más amplio y variado de dimensiones sociales, tales como la gestión del conocimiento, la participación social y la promoción de acciones colectivas orientadas a mejorar la coordinación de los diferentes procesos y la organización de los actores en el nivel productivo local (Klerks et al., 2014). Esto es importante dado que contribuye a visibilizar la manera en que actividades de innovación, entre las que se incluyen la transferencia tecnológica y las capacitaciones, se convierten en un dinamizador de empoderamiento social y de adquisición de nuevos saberes.

Asimismo, conviene repensar el crecimiento inclusivo desde una óptica normativa, a partir de la cual se puedan establecer nuevos parámetros para la evaluación de programas y políticas que tengan como finalidad un desarrollo más equitativo en un marco de sostenibilidad y modernización técnica.

\subsection{Sistemas de innovación: tomando en consideración las transformaciones necesarias}

Como argumentan Cozzenz y Sutz (2012), la literatura que debate la conexión entre la innovación y el desarrollo no es muy extensa, pero cada vez hay más documentos. En este documento sugerimos que al considerar la innovación y los sistemas de innovación como los medios para promover las transformaciones necesarias para alcanzar los objetivos multidimensionales del desarrollo sostenible surge una conexión clara entre innovación y crecimiento inclusivo.

La mayoría de las preguntas clave sobre el estudio de los sistemas de innovación están relacionadas con la explicación de por qué las naciones difieren en cuanto al desempeño económico (Edquist, 1997).

Siguiendo a Malerba (2004), al centrar la atención en sectores específicos, es conveniente seguir un enfoque de sistema de innovación sectorial, estudiando en particular: la base del conocimiento, los actores y las redes, y las instituciones. Es importante colocar el conocimiento y los ámbitos tecnológicos en el centro del análisis. 
De gran relevancia en los enfoques sectoriales son, como lo argumenta Malerba (2005), los procesos de aprendizaje, las competencias, las creencias, los objetivos, las estructuras organizacionales y los comportamientos específicos que caracterizan a cada agente. Con estos enfoques se considera la innovación como un proceso que implica interacciones sistemáticas entre diversos actores para la generación e intercambio de conocimiento relevante para la innovación y su comercialización. Las interacciones incluyen tanto las relaciones de mercado como las no mercantiles, que son más extensas que el mercado de concesión de licencias tecnológicas y conocimiento; las alianzas entre empresas y redes formales de empresas y, a menudo, los métodos existentes para medir la producción económica no capturan sus resultados de manera adecuada.

También son de vital importancia las instituciones, porque el conocimiento, las acciones y las interacciones de los agentes están determinadas por las instituciones, lo que incluye las normas, rutinas, hábitos, prácticas establecidas, reglas, leyes, estándares, etc.

Las relaciones entre las instituciones y las organizaciones en un sistema de innovación influyen en los procesos de innovación y por lo tanto también en el desempeño del sistema (Edquist y Johnson, 1997).

El camino hacia la transformación se centra en los procesos de aprendizaje. Como lo propusieron Lundvall y Lorenz (2010), la teoría detrás del análisis de los sistemas de innovación asume que las organizaciones y los agentes tienen la capacidad de mejorar su competencia mediante el aprendizaje y el descubrimiento, y esto se hace mediante la interacción con otros agentes. Esto se ve reflejado en los procesos de innovación y en los resultados que se manifiestan en nuevas competencias. Se parte además de que el sistema de innovación apoya y determina los esfuerzos y las competencias de las empresas (Nelson, 1998).

\section{Métodos y estudio de caso}

\subsection{Metodología y análisis de la información}

La metodología de este trabajo es predominante cualitativa. Se realiza un análisis sectorial siguiendo la modalidad del estudio del caso. De acuerdo con Yin (1984), un estudio de caso se orienta a investigar y explicar las características de un fenómeno determinado con particular detalle, considerando para ello una descripción, análisis y exploración del objeto de estudio y sus interacciones.

Para la recolección de la información, se realizó una serie de visitas y entrevistas semiestructuradas a actores institucionales y personas vinculadas con el fortalecimiento productivo y la gestión de la innovación en el sector lácteo. De esta manera, se logró reconstruir una visión integral a partir de las valoraciones y reflexiones realizadas por organizaciones públicas, universidades públicas, intermediarios industriales y productores. 
La guía semiestructurada de preguntas que se utilizó está basada en un instrumento utilizado para investigaciones previas relacionadas (Barboza-Arias, 2016), al cual se le agregaron preguntas derivadas de la variable de crecimiento inclusivo.

La información procedente de fuentes secundarias que acá se presenta es el resultado de un análisis documental que se realizó de forma previa a la investigación, para lo cual fueron consultados documentos como el VI Censo Nacional Agropecuario (INEC, 2015).

\subsection{Estudio de caso: la evolución de la industria láctea en Costa Rica}

Como plantea Barboza-Arias (2017), la actividad ganadera en el país está atravesando por una relocalización de usos hacia el doble propósito, lo que sugiere una transformación en la estructura productiva que tiene relación directa con la rentabilidad económica de una actividad más diversificada y en proceso hacia una mayor apertura comercial.

Entre 1984 y 2014, se produjo una disminución de $37.51 \%$ en el hato bovino del país. Si bien el sistema de doble propósito aumentó 28.97 \%, la ganadería en Costa Rica ha experimentado una contracción importante a lo largo de estos años. Este sistema productivo se presenta principalmente en las provincias Alajuela, Heredia, Puntarenas y Limón. La ganadería de carne, en cambio, presenta el declive más pronunciado (56.43\%), mientras que el ganado destinado a la producción leche disminuyó $14.63 \%$ (Gráfico 1).

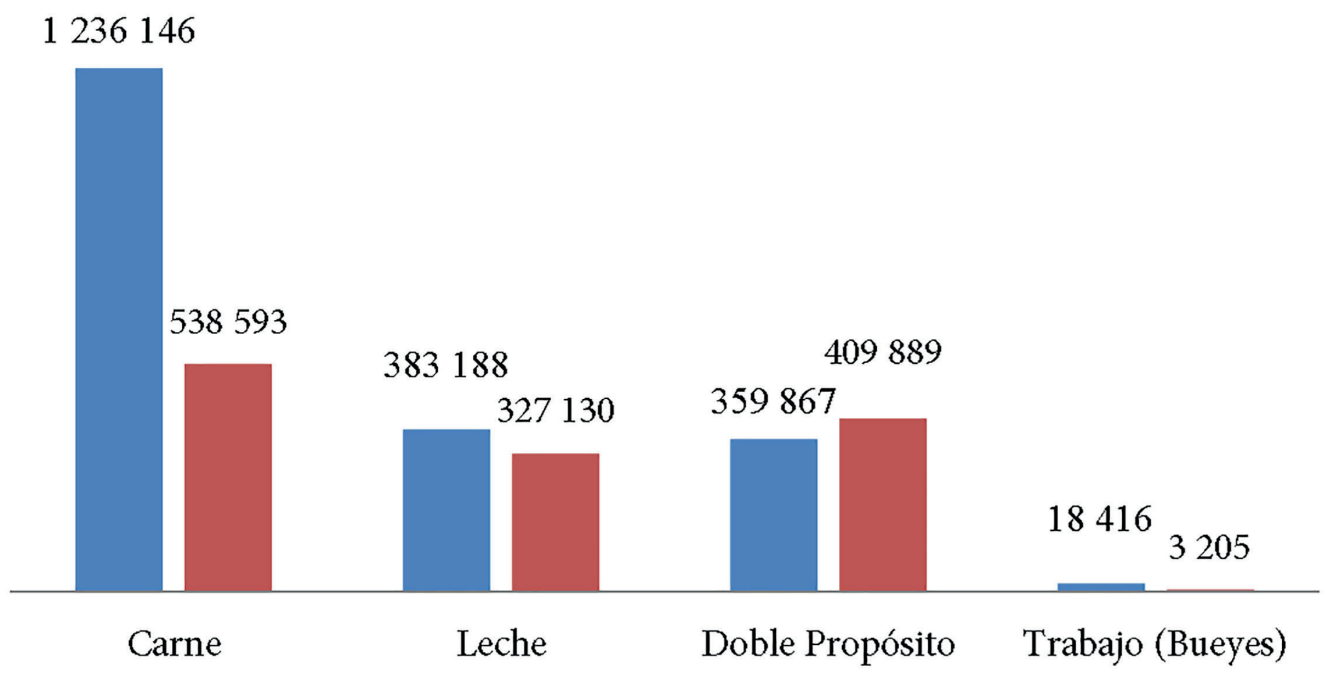

Gráfico 1. Costa Rica: Ganado vacuno por número de animales, según uso. 1984 y 2014.

Fuente: Elaboración propia con base en INEC, 1985 y 2015. 
Los pequeños y medianos productores visualizan el doble propósito como un sistema favorable a la actividad productiva, pues este no solo permite mantener el nivel de rentabilidad económica sino que además estimula la diversificación de productos, como los derivados lácteos de fabricación familiar, y mejores condiciones de acceso a canales de comercialización y nichos locales.

Se trata de una lógica de adaptación productiva que ha estimulado el ingenio y la creatividad de los productores, pues la frugalidad de los recursos y las carencias encontradas con respecto a otros sistemas es resuelta a través de soluciones originales que, si bien pueden considerarse de un alcance restringido, permiten al productor mantenerse en la actividad ganadera sin emigrar a otras actividades económicas.

Abarca $(2015)^{3}$ considera que el sistema de doble propósito emergió como la opción tradicional en la lechería de bajura, mientras que el sistema de lechería especializado se ha continuado implementando en las zonas de altura. El sistema de doble propósito se caracteriza por prácticas empíricas basadas en conocimiento tácito, donde la experiencia cotidiana del productor tiene un peso determinante.

En comparación con el sistema especializado, la lechería de doble propósito presenta deficiencias técnicas e institucionales importantes. La capacidad de absorción tecnológica de los productores pequeños y medianos es obstaculizada por limitaciones importantes en el acceso a recursos financieros y de conocimiento, condición que afecta de manera directa la participación de estos actores en la cadena de valor de láctea y su margen de maniobra en el marco institucional regulado por la estructura organizativa del sector (Barboza-Arias, 2017).

Las razas puras que son utilizadas por la lechería de altura, al no estar genéticamente preparadas para lidiar con las variables climatológicas de las zonas bajas, son afectadas por enfermedades tropicales, presentando también problemas reproductivos y carencias nutricionales debido a la baja calidad de los pastos disponibles.

Abarca (2015) señala que la respuesta inmediata de los productores ha sido la hibridación, cuyo propósito es lograr una mayor resistencia del ganado. Sin embargo, estos "cruces entre especies" presentan la problemática de períodos de lactancia más reducidos y, en consecuencia, la menor producción de leche.

Los productores han optado por prolongar los períodos de lactancia y continuar con las tareas de ordeño, aplicando un esquema rotativo en la manipulación de las ubres, de tal forma que la productividad no decaiga y al mismo tiempo se eviten problemas de salud animal como la mastitis. Por otra parte, se ordeña una sola vez al día para que las vacas puedan pastar junto a las crías más tiempo, reduciendo las cargas de estrés al animal.

3 Comunicación personal. Instalaciones del Instituto Nacional de Innovación y Transferencia en Tecnología Agropecuaria, Ministerio de Agricultura y Ganadería. 2015. 
Esta práctica es común entre los pequeños y medianos productores de las zonas de bajura, quienes basan la alimentación del ganado en concentrado sin alcanzar el rendimiento obtenido por la lechería de altura. Para Abarca (2015), pese a que el sistema de doble propósito es estable y contribuye de manera notable a mantener un nivel alto de producción de leche en Costa Rica, presenta multiplicidad de escenarios de mejora, entre los que destacan: la utilización de razas no definidas (no puras), la carencia de instalaciones adecuadas para la actividad y la falta de pastos de calidad.

No obstante, aún no se han establecido lineamientos específicos para el desarrollo de programas de largo alcance que impulsen mejoras significativas en el desempeño de estos esquemas productivos. En particular, se carece de un diálogo articulado con los actores que permita el diseño de una política sectorial inclusiva, así como de estrategias para promover capacidades de aprendizaje, pensadas desde el punto de vista de la inclusión social y el desarrollo territorial (Barboza-Arias, 2017).

La producción anual de leche alcanzó los 1.135 millones de litros en el año 2016, es decir, más de tres millones de litros diarios. Este dato confirma el aumento sostenido de la producción de leche en el país, que ha permitido además un ligero aumento del consumo nacional y una mayor presencia de productos lácteos en el mercado local y para exportación (Cámara Nacional de Productores de Leche, 2017).

Según los datos producidos por la Cámara Nacional de Productores de Leche (2018), el volumen exportado de productos lácteos de Costa Rica en 2017 fue de 98.37 millones de kilogramos netos, con un valor total de 155.17 millones de dólares (FOB US\$). Se trata de una disminución de menos del $0.01 \%$ en volumen y un aumento del $1.6 \%$ en el valor de exportación en comparación con las cifras del año 2016. Según la misma fuente (2018), el precio por kilo exportado se vio incrementado levemente en un 1.5\% en relación al año anterior, pasando \$1.55 por kilo exportado en 2016 a \$1.58 en 2017.

Además de lo anterior, debe tenerse en cuenta que el mercado regional se caracteriza por un bajo poder adquisitivo y, a nivel global, la tendencia en los mercados parece dirigirse a la sustitución de lácteos por otros productos de origen no animal.

\section{Resultados y discusión}

\subsection{Estructura y actores de la cadena láctea de Costa Rica}

Además de las variaciones en el tratamiento de la producción primaria ocasionadas por la disminución del hato vacuno, la institucionalidad que determina la estructura organizativa 
establece lógicas de integración que son específicas para cada actor dentro de la cadena láctea. De esta manera, las condiciones y procesos que conducen a las actividades de innovación se encuentran determinadas no solo por los cambios potenciales que conducen a la creación de valor agregado sino también por la distribución de poder y los sistemas de control de unos actores sobre otros.

Cabe destacar que pese a los esfuerzos de planificación del sector por parte de las organizaciones vinculadas, en la producción de leche y la industria láctea se percibe una actitud aislacionista que obstaculiza la integración efectiva entre los diferentes segmentos de la cadena de valor.

En la figura 1 se muestra un diagrama de la cadena láctea de Costa Rica. Como se observa, la generación de valor agregado del producto principal, la leche, atraviesa por diferentes etapas antes de llegar al consumidor final. Sin embargo, son los intermediarios industriales y los responsables de la comercialización los actores con mayor influencia sobre el proceso de agregación de valor, dada su capacidad para establecer las condiciones de acceso de la leche al circuito así como su influencia en los gustos y preferencias del consumidor.

Las organizaciones públicas de fomento de la ganadería de leche, así como los centros de investigación, si bien tienen un rol importante en el apoyo al productor, por ejemplo mediante la orientación sobre los protocolos de salud animal y las normativas de inocuidad vigentes, influyen poco en temas de cohesión social y articulación productiva.

De esta manera, los actores privados adquieren un mayor protagonismo en la definición de estrategias de mejora sectorial. Como resultado de ello, a nivel productivo y agroindustrial las cooperativas se han convertido en un agente clave para vincular al productor con los procesos de tecnificación y transferencia tecnológica. A través de estas organizaciones, el asociado tiene acceso a paquetes tecnológicos que le permiten realizar mejoras incrementales en productividad, rentabilidad y sostenibilidad de los sistemas de producción, así como otra serie de beneficios socioeconómicos, incluido el acceso a créditos e insumos de producción. No obstante, los efectos de estos vínculos sobre la producción y, en general, la calidad de vida de los productores no siempre son valorados en términos productivos por quienes acceden a ellos. 


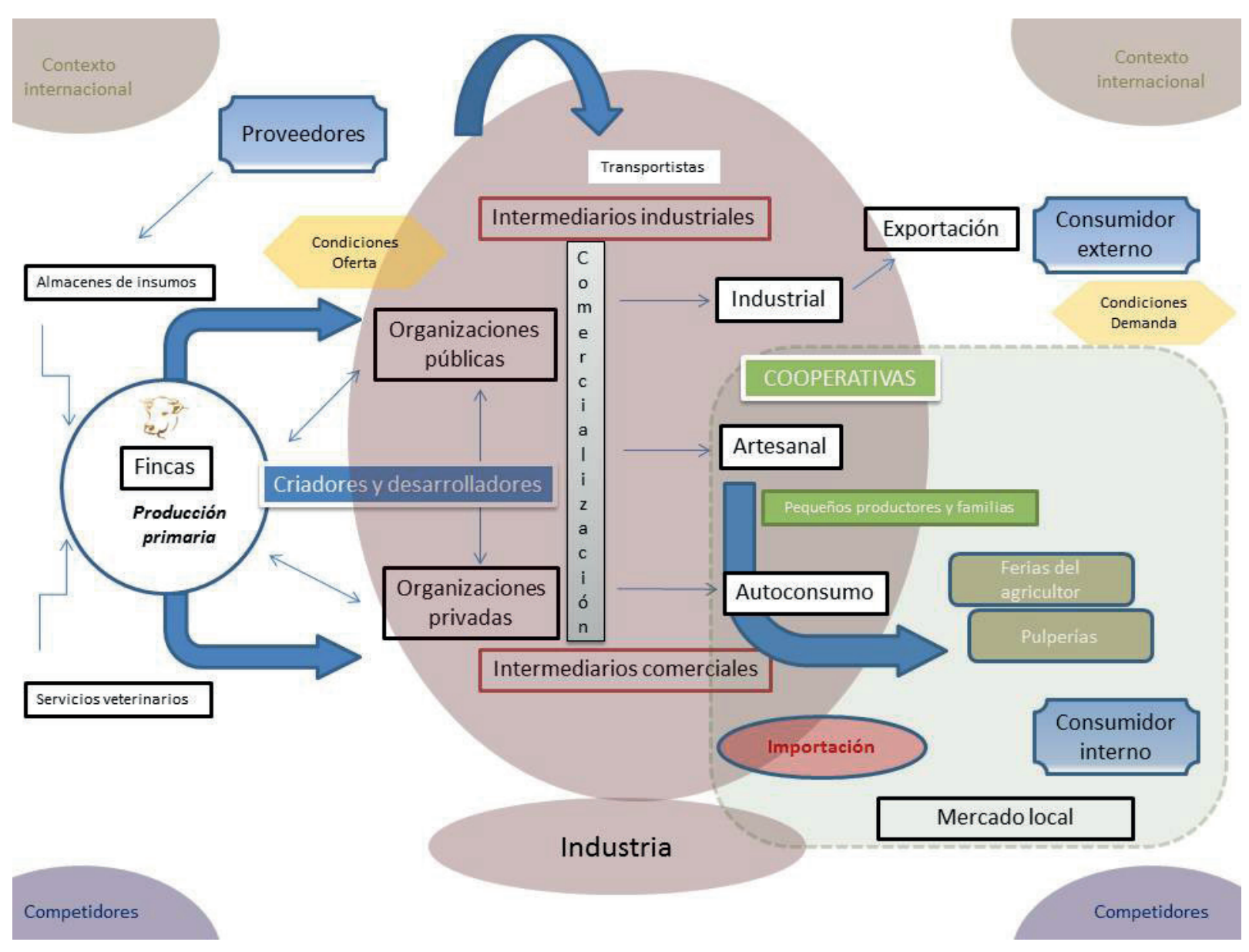

Figura 1. La cadena de valor del sector lácteo de Costa Rica Fuente: Barboza-Arias, 2017.

\section{El rol de las cooperativas en el sector lácteo}

Otro hecho histórico que ha favorecido la evolución del subsector lechero en Costa Rica ha sido el cooperativismo. Existen casos exitosos de cooperativas de productores lecheros que lograron superar diversos obstáculos en el proceso de su constitución organizacional. De acuerdo con León $(2015)^{4}$, las cooperativas distribuyen sus excedentes entre los productores asociados, generándoles estabilidad en el negocio. Existe también una relativa buena oferta de profesionales que trabajan en las cooperativas o en forma independiente, cuya prestación de servicios de apoyo a las fincas ha permitido su evolución a través de la transferencia tecnológica y modernización

4 . Comunicación personal. 2015. 
técnica. En el pasado, cooperativas como Dos Pinos R.L, Coopebrisas y Coopeleche, entre otras, impulsaron estrategias para estimular a agricultores que se dedicaban a actividades agrícolas distintas a incursionar en la ganadería de leche.

Según datos de la Cámara Nacional de Productores de Leche (2015), en 2012 el 88.5 \% de la leche se producía en fincas de productores asociados a cooperativas o miembros de asociaciones de productores. Actualmente, la Cooperativa Dos Pinos, conformada por aproximadamente 1.400 socios, recoge alrededor del $80 \%$ de la leche que ingresa al circuito industrial.

Desde el punto de vista de crecimiento inclusivo, el aporte de las cooperativas ha sido significativo en tanto ha permitido que un amplio número de pequeños productores tenga acceso a tecnología de punta para la producción y manejo de la leche, así como acceso a ganancias de la industrialización de esta.

\section{Reglas del juego en el marco de los tratados de libre comercio}

Un aspecto de relevancia para el sector es que en el año 2016 entró en vigor la desgravación arancelaria acordada por Costa Rica en el marco de las rondas de negociación del Tratado de Libre Comercio entre Estados Unidos, Centroamérica y República Dominicana.

En ese marco, se establece la apertura comercial para la leche y los derivados lácteos. Para Abarca (2015), se trata de un nuevo punto de quiebre en la evolución histórica del subsector, mientras que León (2015) lo considera una preocupación permanente, dado el alto costo de la producción de leche en territorio nacional, el alto costo de los insumos importados y las altas cargas sociales que nuestros productores deben pagar, todo ello inmerso en un contexto internacional caracterizado por la alta inestabilidad de los precios internacionales de la leche.

Desde el punto de vista de crecimiento inclusivo, es claro que los pequeños productores se enfrentan a grandes retos para mantenerse competitivos dentro de un mercado cada vez más expuesto a la competencia internacional. El reto también lo tienen las cooperativas y las entidades estatales que deberán reforzar los esquemas de investigación y desarrollo, así como los mecanismos de transferencia tecnológica, que permitan sobrellevar esa creciente apertura de mercados.

\subsection{Estrategias de acción para la innovación y el desarrollo inclusivo del subsector lechero en Costa Rica}

En la cadena de valor de la leche y los derivados lácteos participan un conjunto variado de actores: productores, proveedores e industria convergen en la dinámica productiva del subsector lechero, en la que interviene además el Estado (a través de las organizaciones públicas competentes), organizaciones privadas (como la Cámara Nacional de Productores de Leche) y los Centros de Investigación y Transferencia de Tecnología.

Dada la composición del sector, la producción de leche en Costa Rica se canaliza por medio de tres circuitos: el industrial, compuesta por una variedad de cooperativas y plantas de capital 
privado, el informal o artesanal, y el autoconsumo. De acuerdo con Abarca (2015), la actividad lechera de tipo industrial se encuentra conformada actualmente por la Cooperativa Dos Pinos R.L., que recoge alrededor del $80 \%$ de la leche que ingresa al circuito industrial (el $50 \%$ de la leche total que se produce en el país); dos industrias con capacidad de operación más reducida: Sigma y Coopeleche, y algunas otras pequeñas que funcionan a nivel local. Un aspecto de suma relevancia que determina la situación actual del sector es un contexto nacional caracterizado por una industria en evolución, con la presencia de un mayor número de empresas medianas y artesanales. Para el año 2016, existían 8 empresas de escala mediana y 120 procesadores pequeños.

La lechería de tipo artesanal, que realizan en su mayoría los microproductores en plantas procesadoras de tradición y capital familiar, se comercializa a través de terceros (intermediarios), o nichos de mercado pequeños, como Ferias del Agricultor y pulperías locales. Un grupo reducido de pequeños productores realiza además la venta al por menor de sus productos: leche fluida y quesos frescos en su mayoría, que son por lo general producidos en sus propias fincas en condiciones desfavorables.

La actividad lechera está dominada por grandes y medianos productores, pues el micro y pequeño productor no tienen opciones de formar parte de los centros de acopio, por la exigencia de aportes en certificados de inversión, cuotas fijas de entregas de leche y otra serie de condiciones que evitan la participación.

Por lo tanto, la mayoría de los pequeños y medianos productores de leche de bajura operan de forma independiente (Abarca, 2015), lo cual se convierte en un obstáculo al desarrollo inclusivo del subsector. Sin embargo, las cooperativas y gremios organizados de productores forman el pilar de la actividad. A través de la asistencia técnica que brindan estas organizaciones los productores asociados han logrado mejorar sus capacidades y adquirir nuevos conocimientos y destrezas.

Otros servicios, como los créditos en compras que luego son descontados de sus cheques por entregas de leche y otra serie de beneficios, se traducen en una mejor calidad de vida y un grado mayor de inclusión social. Para la industria, la participación activa de los asociados se traduce en el fortalecimiento de las actividades económicas paralelas a la producción, permitiendo de esta manera la atención integral de las necesidades del productor. La captación de recursos financieros facilita, además de la gestión de la producción y el modelo de negocios, la obtención de los insumos y bienes requeridos por el productor para la industrialización de la leche.

En la actualidad, la Cooperativa Dos Pinos R.L. lidera los procesos de innovación, investigación y desarrollo de la actividad de leche industrial. Con niveles de inversión altos, se ha enfocado en la eficiencia y la competitividad del sector productivo a través de recursos para 
el apoyo técnico y la investigación de la mano de las universidades y entes de investigación y desarrollo tecnológico, entre otros. El $100 \%$ de las fincas de productores asociados cuentan con equipo de ordeño y un tanque de enfriamiento para la leche, que se recolecta en camiones modernos, garantizando de esta forma la inocuidad del producto.

Para Zamora $(2015)^{5}$, uno de los principales beneficios de la relación cooperativaproductor es la mejora continua en las fincas, cuyos índices de productividad y calidad de la leche aumentan. Esta empresa encabeza además la industria láctea de gran escala, liderando los procesos de calidad a través de la tecnificación y la modernización tecnológica. Cuenta en sus instalaciones con departamentos formales de innovación e investigación y desarrollo, con lo cual se enfoca en dar un mayor valor agregado a la leche a través de productos diferenciados.

Los procesos de producción y calidad de Dos Pinos se han convertido en un referente de benchmarking para las industrias del subsector. Por ejemplo, Sigma Alimentos, que después de adquirir Monteverde y Lekerland recibe la leche de aproximadamente 600 productores (CNPL, 2015), ha tratado de incorporar como parte de su estrategia servicios de veterinaria, agronomía y asistencia a la finca, similares a los implementados por Dos Pinos (Cruz, 20156).

La ventaja competitiva de Dos Pinos sobre otras industrias del subsector se basa en el valor agregado del conocimiento de sus productores, alcanzado en parte por el Programa de Transferencia Tecnológica de la Cooperativa, que dispone de una buena oferta de profesionales que trabajan de la mano con el productor, brindando capacitaciones en procesos básicos como el uso apropiado de ordeños mecánicos y los equipos de refrigeración transferidos a la finca, como los tanques de enfriamiento.

A pesar de su balance positivo, Abarca (2015) considera que el nicho tradicional de Dos Pinos R.L. han sido los productores de altura y, en los últimos años, la empresa se ha mostrado poco anuente a expedir nuevos certificados asociativos. Eso genera una limitante para el crecimiento inclusivo, pues el esquema que históricamente ha dado acceso a muchos pequeños productores comienza a dejar de ser una opción para nuevos productores.

Si se consideran los niveles de integración en los que podría clasificarse la actividad lechera (figura 2), es posible identificar que esta restricción repercute de forma directa sobre nuevas lógicas de exclusión. Al operar de forma independiente, los productores no solo quedan fuera del marco institucional de la cadena láctea sino también de las opciones formales de capacitación, flujos de información y gestión de nuevos conocimientos para promover el desempeño innovador.

5 Comunicación personal. Instalaciones de Cooperativa de Productores de Leche Dos Pinos R.L. 2015.

6 . Comunicación personal. Visita a Lechería en Cartago. 2015. 


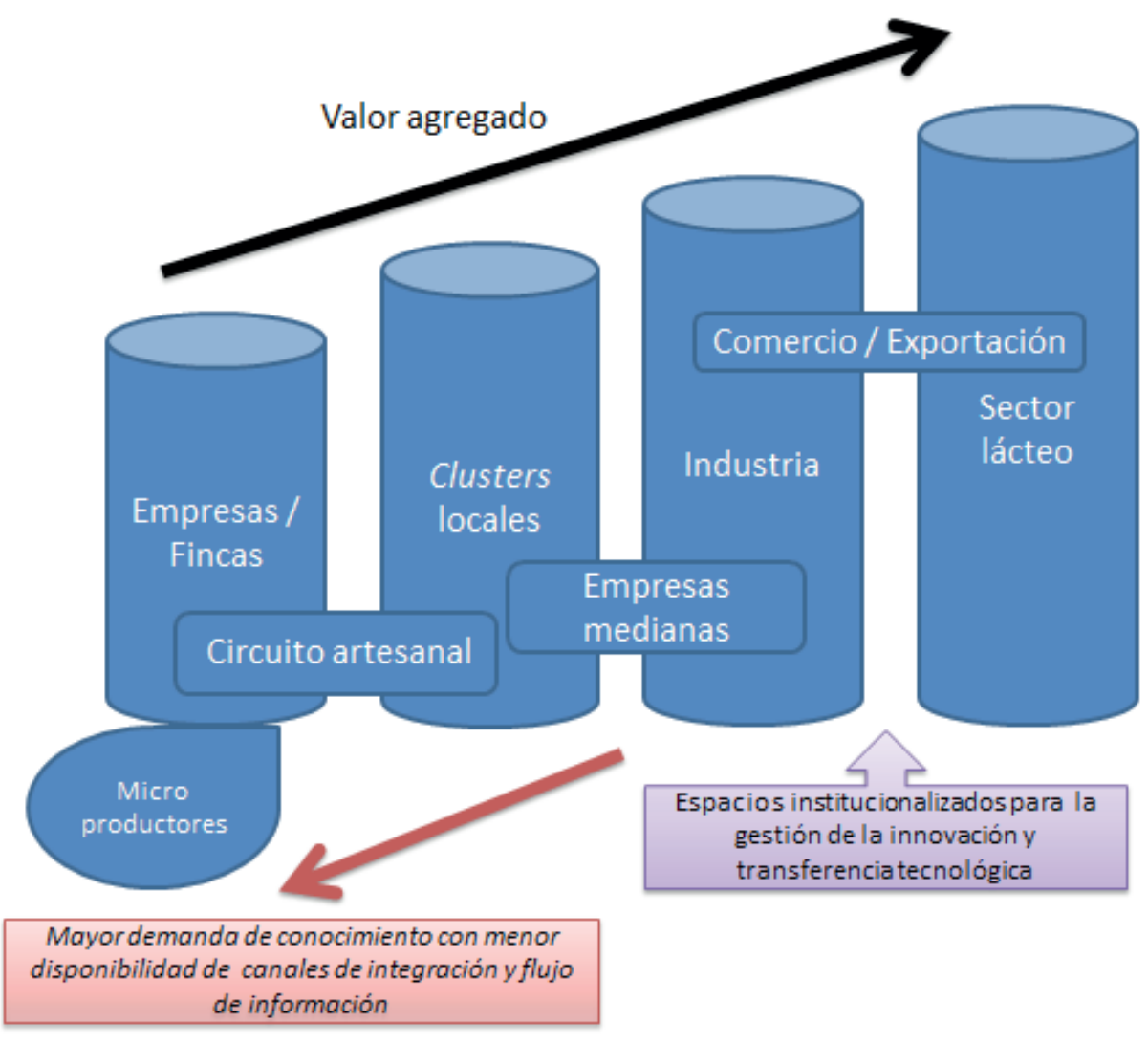

Figura 2. Niveles de integración de la cadena láctea y gestión de la innovación en el sector lácteo de Costa Rica Fuente: Elaboración propia, 2017.

\subsection{Promoción de la innovación y competitividad del subsector lechero en Costa Rica}

En el plano productivo, la planificación de procesos para la innovación es resultado de un conjunto de dinámicas históricas y organizacionales fuertemente influenciadas por la participación de las cooperativas y sus objetivos de desarrollo productivo. Es posible identificar al menos tres ejes prioritarios para la gestión de la innovación y la competitividad del sector: mejoramiento genético, nutrición y manejo de pasturas.

\section{Mejoramiento genético}

Desde el INTA, el principal objetivo de la estrategia de mejoramiento genético es promover la investigación nacional y la apropiación de esta por parte de los productores de 
Revista de Política Económica • EISSN: 2215-4167

Vol. 4 (1) • Julio-Diciembre, 2018: 1-20

DOI: http://dx.doi.org/10.15359/peds.4-1.2

Orozco-Barrantes y Barboza-Arias

URL: http://www.revistas.una.ac.cr/politicaeconomica

leche locales. Mediante la transferencia tecnológica y las iniciativas de modernización técnica, se da continuidad a la adaptación del sistema de producción al clima cálido de las zonas de bajura. Mediante procesos de $\mathrm{I}+\mathrm{D}$, se busca mejorar la producción y calidad de pastos y forrajes, con el propósito de lograr el balance nutricional del hato y disminuir la dependencia de los concentrados elaborados a base de granos. Estudios científicos han demostrado que los pastos producidos en zonas subtropicales son altamente nutritivos (Abarca, 2015); sin embargo, su estructura fisiológica hace imposible su traslado directo. La estrategia tecnológica del INTA, ante esta disyuntiva, es la importación de semen de ganado proveniente de zonas calientes, como Brasil. En la actualidad, existen mecanismos legales para este tipo de importación, pero sus costos son elevados. Además, al ser Brasil un país considerado como aftoso, el uso de este semen traería consecuencias negativas a la exportación de leche costarricense hacia mercados desarrollados, como Estados Unidos y Europa.

Se trabaja también en la identificación de un esquema organizacional para el fomento de la inseminación artificial, que permita al mismo tiempo su distribución entre los productores, en particular los pequeños y medianos; así como una estrategia de producción estacional que permita a los productores de leche dedicarse a otras actividades productivas durante la temporada lluviosa. Se trata, en todo caso, de acciones pendientes que requieren de la capacidad de extensión de las organizaciones del Estado y la vinculación con el sector privado.

\section{Mejora nutricional/alimenticia}

La importación de variedades de pasto durante la década de los noventa favoreció el ingreso al país de germoplasma de pasto de bajura. Sin embargo, este no tuvo los efectos esperados debido a que fue utilizado para alimentar las razas del sistema especializado de las zonas de altura, generando ineficiencias en la alimentación y la productividad del ganado. Por otra parte, los problemas asociados a la pérdida de escala de la producción de leche en el país, provocada en los últimos años por la tendencia al fraccionamiento de las fincas en unidades productivas más pequeñas, tuvieron consecuencias negativas en el sistema de alimentación a base de pastoreo.

Por otra parte, la producción de forrajes es otra de las áreas en donde se están produciendo mejoras. Se ha aumentado el consumo de materia seca en las fincas a partir de la implementación del sistema de semiconfinamiento, cuyo balance nutricional permite aumentar la producción de leche. La incorporación de este sistema está además acompañada de cambios organizacionales, ya que se requiere la construcción de sistemas especiales de alojamiento, equipo y tecnologías para lograr las condiciones óptimas que permitan preservar las propiedades del forraje. Actualmente se trabaja en conjunto con productores en la mecanización de la cosecha de forrajes y en formas eficientes de almacenamiento (CNPL, 2015).

Las organizaciones del sector colaboran con los productores, apoyándoles en el proceso de conversión de un sistema de pastoreo a sistema semiestabulado. Entre otros beneficios de la semiestabulación, se encuentran: la mitigación del efecto del clima sobre el animal, la optimización 
Revista de Política Económica • EISSN: 2215-4167

Vol. 4 (1) • Julio-Diciembre, 2018: 1-20

DOI: http://dx.doi.org/10.15359/peds.4-1.2

Orozco-Barrantes y Barboza-Arias

URL: http://www.revistas.una.ac.cr/politicaeconomica

del consumo de materia seca a través de la lactancia, la vida útil de las pasturas se ve favorecida a la vez que se aprovechan las excretas como sustitución parcial de la fertilización, se reducen las variaciones extremas en entregas de leche así como problemas reproductivos en el animal, si bien de lo último aún no se dispone de evidencia. Por último, el sistema semiestabulado ayuda a mantener, e incluso aumentar, la carga animal de la finca. En concreto, con la incorporación del sistema semiestabulado se logra una mejora en el balance energético, la producción leche y la condición corporal del animal.

No obstante, el cambio de un sistema a otro, como cualquier adaptación, es también un proceso de enseñanza-aprendizaje en el que se debe potenciar las capacidades de los productores que lo llevan a cabo. La asesoría institucional, así como el seguimiento técnico, son criterios a tener en cuenta por parte de las instituciones pues, como los expertos indican, el cambio de sistemas productivos requiere mayor control y prevención administrativa (Rojas, 2016).

\section{Manejo de pasturas}

La capacidad de manejo y almacenamiento de pastos y forrajes es una de las áreas críticas en la que las innovaciones institucionales tienen menor incidencia. En consecuencia, el impacto en la inclusión social de pequeños y medianos productores también ha sido inferior. Si bien la importación de pastos favoreció el ingreso al país de variedades nuevas, Abarca (2015) reconoce que no hubo una estrategia de capacitación efectiva que permitiera a los productores asesorarse en el manejo de los pastos y forrajes. La importación continua de semillas por parte de los productores incrementa los costos de producción y pese a que su siembra se estima para períodos de 10 años, los productores costarricenses realizan re-siembras cada dos años en promedio, lo cual hace que esta actividad no resulte rentable y genere costos de ineficiencia. La importación de semillas, sin embargo, es una práctica realizada solamente por productores de altura. El sistema de doble propósito enfrenta además problemas relacionados con el manejo de las vacas, cuyos niveles de producción por animal no alcanzan el rendimiento definido por los estándares internacionales.

La lechería de bajura debe adaptarse a la mala calidad de los suelos, que influye en la productividad de los pastos. La zona de ganadera de Turrialba ha sido una de las regiones más afectadas por estos factores. Suelos suaves y períodos de lluvia prolongados provocan que el crecimiento natural del pasto resulte alterado. La producción de leche en Turrialba se caracteriza por su empirismo y la prevalencia de un sistema artesanal anclado fuertemente en la experiencia subjetiva del productor, lo cual provoca un rezago tecnológico que se evidencia en la recarga animal por hectárea y la falta de soluciones integrales para superar la escasez de pastos. A través del apoyo interinstitucional y el acompañamiento a productores locales que realizan diversos centros de investigación como el Catie, se trabaja con productores y sus familias en la creación de competencias y capacidades. La vinculación se establece a través de la Asociación de Productores Agropecuarios de Santa Cruz (Asoproa) y ha favorecido a los 250 productores que integran la Asociación en la actualidad, logrando cambios cualitativos a través de redes de trabajo y 
Revista de Política Económica • EISSN: 2215-4167

Vol. 4 (1) • Julio-Diciembre, 2018: 1-20

DOI: http://dx.doi.org/10.15359/peds.4-1.2

Orozco-Barrantes y Barboza-Arias

URL: http://www.revistas.una.ac.cr/politicaeconomica

mejoras organizacionales en la unidad productiva, entre ellas, la construcción de invernaderos y biodigestores, cuya implementación permitió reducir los niveles de contaminación en las fincas, y el manejo de estiércol para la producción de abono orgánico: actividades orientadas a la implementación de un modelo de lechería ecoeficiente y sostenible.

Además, desde la Cámara Nacional de Productores de Leche se impulsa la Red Nacional de Forrajes, una iniciativa dirigida a generar y transferir tecnologías para la producción y utilización de forrajes y otros alimentos a los productores para mantener la competitividad y sostenibilidad de los sistemas de producción leche, doble propósito y carne, del país. Se trata de una plataforma de apoyo al Programa de Investigación y Transferencia de Tecnología Agropecuaria (Pitta), derivado de la Estrategia Nacional de Ganadería Baja en Carbono.

\section{Conclusiones y recomendaciones de política}

A lo largo del tiempo se han generado algunas innovaciones institucionales que contribuyeron al crecimiento inclusivo del sector lácteo. Sin embargo, las reglas del juego han ido cambiando y se ha ido propiciando un esquema cada vez menos inclusivo.

La exposición de los productores a la apertura comercial, especialmente con la entrada en vigencia del proceso de desgravación pactado en el marco del Tratado de Libre Comercio entre Centro América con Estados Unidos y República Dominicana, genera grandes retos. La desgravación arancelaria para los productos lácteos se reduce de forma paulatina desde el año pasado y llegará a 0 en el 2025, lo cual, sin duda alguna, tendrá efectos sobre la producción nacional que aún se desconocen.

Conceptualmente, el Estado costarricense parece tener claro que el esquema americano de leche de altura no es el mejor en latitudes más bajas y que los diferentes programas de apoyo a la ganadería son ejemplo de ello. Sin embargo, no es claro que la estrategia propuesta integre todos los componentes para que el sistema sectorial como un todo funcione apropiadamente.

Para mejorar el funcionamiento del sistema sectorial de innovación, es importante integrar el esfuerzo de múltiples actores. Un buen ejemplo es el esquema de vinculación logrado por la Asociación de Productores Agropecuarios de Santa Cruz (Asoproa), que ha favorecido a los 250 productores que integran la Asociación en la actualidad, logrando cambios cualitativos a través de redes de trabajo y mejoras organizacionales en la unidad productiva, entre ellas, la construcción de invernaderos y biodigestores, cuya implementación permitió reducir los niveles de contaminación en las fincas y el manejo de estiércol para la producción de abono orgánico, y que, en general, apuntan a la implementación de un modelo de lechería ecoeficiente y sostenible.

Las cadenas de producción, dominadas por actores grandes, han generado impactos positivos en los miembros de ese tipo de asociaciones, pero generan limitaciones para los productores que están fuera. Es necesario, por tanto, reforzar los mecanismos de asociatividad 
Revista de Política Económica • EISSN: 2215-4167

Vol. 4 (1) • Julio-Diciembre, 2018: 1-20

DOI: http://dx.doi.org/10.15359/peds.4-1.2

Orozco-Barrantes y Barboza-Arias

URL: http://www.revistas.una.ac.cr/politicaeconomica

en nuevos esquemas que den acceso al financiamiento y a los paquetes tecnológicos que permitan un crecimiento con oportunidades para pequeños productores.

En el nivel de la coordinación sectorial, existen vetas importantes a la participación efectiva de los productores en los circuitos locales de comercialización y su escalonamiento en el resto de la cadena. No obstante, se puede identificar que estas oportunidades de mejora pueden tener un mayor potencial y contribuir al crecimiento inclusivo del sector si son atendidas desde las propias organizaciones de productores, con el apoyo efectivo de las estructuras de apoyo, incluyendo las organizaciones del mesonivel como la Corporación Ganadera (CORFOGA) y las instituciones de fomento agroproductivo que conforman el Ministerio de Agricultura y Ganadería (MAG).

En este punto, queda claro que la gobernanza del sistema de innovación del sector lácteo debe responder a un sistema de alianzas público-privadas, a partir del cual se promueva una mejor articulación entre los distintos segmentos de la cadena. Desde el punto de vista de la inclusión social, las organizaciones de productores, las cooperativas encargadas de procesos agroindustriales y las instituciones públicas que participan en los procesos de innovación adquieren una mayor relevancia en la medida en que estos actores presenten la capacidad para promover una institucionalidad vigorosa, más acorde con la dinámica socio-productiva del sector y orientada a la atención de demandas que superan el enfoque tecnocrático.

Cualquier esfuerzo de carácter institucional o apoyado desde la política pública para estimular la innovación y el conocimiento en la cadena láctea debe corresponderse con los objetivos de la política de desarrollo nacional, contener una perspectiva del desarrollo territorial y dialogar de modo reflexivo con los diferentes actores que intervienen en la gestión del aprendizaje y el establecimiento de sinergias para la acción colectiva y la participación inclusiva de los productores en un modelo integrador.

La innovación es un proceso en el cual el desarrollo tecnológico aparece combinado con nuevas formas de organización y acuerdos institucionales emergentes (Yang et al., 2014). Esta combinación implica la aparición de nuevas formas y tipos específicos de coordinación entre actores a través del establecimiento de redes de innovación. Por lo tanto, es importante que, desde una perspectiva de política económica, se identifiquen los mecanismos de evaluación que resultan necesarios para determinar el efecto de los programas de gestión de la innovación en el establecimiento de redes funcionales. Se debe trabajar además en una revisión más atenta de los canales a través de los cuales circulan los flujos de conocimiento e información que demandan los actores involucrados.

Otros elementos a los que el instrumental de la política económica puede contribuir enormemente son la mejora de la capacidad organizacional, el diseño de políticas asertivas, la infraestructura, el financiamiento y la construcción de mercados competitivos. No obstante, para ello resulta pertinente profundizar en la construcción de indicadores que permitan identificar cuál ha sido el nivel de alcance logrado y cuáles son aquellas áreas críticas que requieren de una intervención prioritaria. 
Revista de Política Económica • EISSN: 2215-4167

Vol. 4 (1) • Julio-Diciembre, 2018: 1-20

DOI: http://dx.doi.org/10.15359/peds.4-1.2

Orozco-Barrantes y Barboza-Arias

URL: http://www.revistas.una.ac.cr/politicaeconomica

\section{Referencias}

Ali, I. \& Zhuang, J. (2007) Inclusive Growth toward a Prosperous Asia. Policy Implications. ERD Working Paper Series, 97. Economics and Research Department, Asian Development Bank, Manila.

Arocena, R. y Sutz, J. (2002). Sistemas de Innovación y Países en Desarrollo. Universidad de la República de Uruguay. [Electronic version]. SUDESCA Research Papers 30.

Arond, E., Rodríguez, I., Arza, V., Herrera, F., \& Sánchez, M. (2011). Innovation, sustainability, development and social inclusion: Lessons from Latin America. STEPS Working Paper 48. Brighton: STEPS Centre.

Barboza-Arias, L. M. (2016). Creación de competencias y capacidades en el subsector lechero costarricense. Revista de Política Económica para el Desarrollo Sostenible, 2 (1), pp. 1-28.

Barboza-Arias, L. M. (2017). Fortalecimiento competitivo y organizacional de la ganadería de carne en Costa Rica: propuesta de mejora para la Corporación Ganadera (Corfoga). Cuadernos de Política Económica. Heredia: CINPE, UNA.

Bijman, J. \& G. Ton (2008) Producer organisations and value chains. Capacity.org (34), pp. 4-6.

Cámara Nacional de Productores de Leche (CNPL) (2015). Documentos técnicos. Recuperado de http://www.proleche.com/

Chaminade, C., Lundvall, B. A., Vang, J., \& Joseph, K. J. (2009). Designing innovation policies for development: towards a systemic experimentation based approach. In Lundvall, B. et al. (Eds.). Handbook on Innovation Systems and developing Countries: Building Domestic Capabilities in a Global Setting. United Kingdom: Edward Edgar.

Cozzens, S. \& Sutz, J. (2012). Innovation in Informal Settings: A Research Agenda. Program on Innovation for Inclusive Development (IID) of the Canadian International Development Research Centre (IDRC).

Edquist, C. (1997). Systems of innovation approaches-their emergence and characteristics. In Fagerbert, J. Mowery, D. C. \& Nelson, R. R. (Eds.). System of innovation: technologies, institutions and organizations. London, England: Oxford University Press Pinter.

Edquist, C. \& Johnson, B. (1997). Institutions and organisations in systems of innovation. In Edquist, C. (Ed.) Systems of Innovation: Technologies, Institutions and Organizations. London and Washington: Pinter/Cassell Academic.

Gereffi, G., \& Lee, J. (2016). Economic and social upgrading in global value chains and industrial clusters: Why governance matters. Journal of Business Ethics, 133(1), pp. 25-38. Doi:10.1007/ s10551-014-2373-7 
Revista de Política Económica • EISSN: 2215-4167

Vol. 4 (1) • Julio-Diciembre, 2018: 1-20

DOI: http://dx.doi.org/10.15359/peds.4-1.2

Orozco-Barrantes y Barboza-Arias

URL: http://www.revistas.una.ac.cr/politicaeconomica

Instituto Nacional de Estadística y Censos (INEC). (1985). V Censo Nacional Agropecuario. San José: INEC.

Instituto Nacional de Estadística y Censos (INEC). (2015). VI Censo Nacional Agropecuario. San José: INEC.

Kaplinsky, R. (2015). Global value chains in manufacturing industry: Where they came from, where they are going and why this is important. In Weiss, John/Tribe, Michael (eds): Routledge Handbook of Industry and Development. London: Francis \& Francis Group, pp. 184-203.

Klerkx, L., Aarts, N., \& Leeuwis, C. (2010). Adaptive management in agricultural innovation systems: The interactions between innovation networks and their environment. Agricultural systems, 103(6), 390-400.

Lundvall, B. y Lorenz, E. (2010). Innovación y desarrollo de competencias en la economía del aprendizaje. Implicaciones para las políticas de innovación. En Parrilli, M. (Ed.). Innovación y aprendizaje: lecciones para el diseño de políticas. España: Innobasque, 44-99.

Malerba, F. (Ed.). (2004). Sectoral systems of innovation: concepts, issues and analyses of six major sectors in Europe. Camvridge, UK: Cambridge University Press.

Malerba, S. (2005). Sectoral Systems: How and why innovation differs across sectors. In Fagerberg J, Mowrey, D and Nelson R. (Eds.). The Oxford Handbook of Innovation, pp. 380- 406, Oxford: Oxford University Press, Oxford.

Nelson, R. R. (1998). National innovation systems. Regional Innovation, Knowledge and Global Change. London: Pinter.

Orozco, J. (2004). Innovation and Performance Improvements in the Cooperative Sector, Costa Rica. SUDESCA Research Papers No. 38, Aalborg University, CINPE-UNA.

Rojas, A. (2016). Consideraciones técnicas en la escogencia del sistema de producción: pastoreo - semiestabulación-estabulación. Memorias del Congreso Lechero. Costa Rica: Cámara Nacional de productores de Leche.

Sampedro, J. L. y Díaz, C. (2016). Innovación para el desarrollo inclusivo: Una propuesta para su análisis. Economía Informa, (396), pp. 24-48

Van den Bergh, J. C. (1996). Ecological economics and sustainable development. Theory, methods and applications. Northampton, MA, USA: Edward Elgar Publishing Ltd.

Yin, R. K. (1984). Case Study Research: design and Methods, Applied social research. Methods Series, Newbury Park CA: Sage. 